Canad. Math. Bull. Vol. 23 (1) 1980

\title{
MINIMALLY GENERATED MODULES
}

\author{
BY
}

W. H. RANT

\begin{abstract}
A non-zero module $M$ having a minimal generator set contains a maximal submodule. If $M$ is Artinian and all submodules of $M$ have minimal generator sets then $M$ is Noetherian; it follows that every left Artinian module of a left perfect ring is Noetherian. Every right Noetherian module of a left perfect ring is Artinian. It follows that a module over a left and right perfect ring (in particular, commutative) is Artinian if and only if it is Noetherian. We prove that a local ring is left perfect if and only if each left module has a minimal generator set.
\end{abstract}

In this paper rings have unity and modules are unitary.

It is well-known that any non-zero finitely generated module contains a maximum submodule.

THEOREM 1. If a module $M \neq\{0\}$ has a minimal generator set then $M$ contains a maximum submodule, and hence $M \neq \operatorname{Rad} M$.

Proof. Let $\left\{m_{i}\right\}$ be a minimal generator set for $M$. The submodule $T$ generated by $\left\{m_{i} \mid i \neq j\right\}$ is proper. By Zorn's lemma there is a maximal proper submodule of $M$ containing $T$.

Theorem 2. Let $N_{0}=M, N_{i+1}=\operatorname{Rad} N_{i}$. If $M$ is Artinian and if each $N_{i}$ (in particular, if each submodule of $M$ ) has a minimal generator set, then $M$ is Noetherian.

Proof. Since $N_{i}=N_{i+1}=\operatorname{Rad} N_{i}$ for some $i, N_{i}=\{0\}$ by Theorem 1. Since $\operatorname{Rad}\left(N_{\mathrm{p}} / N_{\mathrm{p}+1}\right)=\{0\}$ and $N_{\mathrm{p}} / N_{\mathrm{p}+1}$ is Artinian, $N_{\mathrm{p}} / N_{p+1}$ has finite length, and since $N_{i}=\{0\}$, we have a composition series for $M$, so $M$ is Noetherian.

THEOREM 3. Every left module of a left perfect ring has a minimal generator set, so every left Artinian module is Noetherian.

Proof. Let $M$ be a left $A$-module and let $R=\operatorname{Rad} A$. Since $A / R$ is semisimple, $M / R M$ is a direct sum of simple modules and so it has a minimal generator set $\left\{m_{j}+R M\right\}$. Clearly $\left\{m_{i}\right\}$ is a minimal generator set for the module it spans, $N$. Since $M=N+R M, M / N=R(M / N)$, and since $R$ is left $T$ nilpotent, $M / N=\{0\}$ (see 1 , Lemma 2.6, p. 473), so $M=N$ and $M$ has a

Received by the editors May 15, 1978 and, in revised form, November 27, 1978.

AMS 1972 subject classifications. Primary 1610, 1640; Secondary 1650. Key words and phrases. Minimal generator set, perfect ring, Noetherian module, Artinian module, socle, injective module, finitely generated. 
minimal generator set. From Theorem 2 every left Artinian module is Noetherian.

LEMMA 1. If $M$ is a Noetherian module and all non-zero quotient modules of $M$ have non-zero socles, then $M$ is Artinian.

Proof. Let $S_{0}=\operatorname{Soc} M$ and $S_{i+1} / S_{i}=\operatorname{Soc}\left(M / S_{i}\right)$. Since $S_{1} \subseteq S_{2} \subseteq \ldots$, we have $S_{k}=S_{k+1}$ for some $k$. Since $\operatorname{Soc}\left(M / S_{k}\right)=S_{k+1} / S_{k}=\{0\}$ and non-zero quotient modules have non-zero socles, we have $M=S_{k}$. Now $S_{1} / S_{0}, S_{2} / S_{1}, \ldots, M / S_{k-1}$ are semi-simple, and since $M$ is Noetherian, they are finitely generated so they have finite length. Thus we have a composition series for $M$, hence $M$ is Artinian.

THEOREM 4. Every right Noetherian module of a left perfect ring is Artinian.

Proof. From [1, Theorem $P$ ] each right module of a left perfect ring has a non-zero socle.

Corollary 1. A module of a left and right perfect ring is Artinian if and only if it is Noetherian.

THEOREM 5. A local ring is left perfect if and only if each left module has a minimal generator set.

Proof. If $A$ is left perfect each left module has a minimal generator set by Theorem 3. For the converse, let $M$ be a flat left module with minimal generator set $\left\{m_{i}\right\}$. Let $F$ be a free module with $\left\{x_{i}\right\}$ as a basis, and let $K$ be the kernel of the homomorphism from $F$ to $M$ sending $\sum a_{i} x_{i}$ to $\sum a_{i} m_{i}$. If $k=\sum a_{i} x_{i} \in K$, then $\sum a_{i} m_{i}=0$; and since $\left\{m_{i}\right\}$ is minimal, each $a_{i}$ is a non-unit and so $a_{i} \in R$, the maximal ideal of $A$; Thus $K \subseteq R F$. Now $M \approx F / K$, and since $M$ is flat, $R F \cap K=R K$, and since $K \subseteq R F, K=R K$. If $\left\{k_{i}\right\}$ is a minimal generator set for $K$, since $K=R K, k_{i}=\sum r_{i} k_{j}$ for some $r_{j} \in R$, so $k_{i}=$ $\left(1-r_{i}\right)^{-1} \sum_{j \neq i} r_{j} k_{j}$. Since $\left\{k_{i}\right\}$ is minimal we have $r_{j}=0$ if $j \neq i$, so $k_{i}=0$, thus $K=\{0\}$ and $M$ is free. From [4, Theorem 1] $R$ is left $T$-nilpotent, so $A$ is a left perfect ring.

Corollary 2. A commutative ring is perfect if and only if each left module has a minimal generator set.

Proof. Theorem 3 and Theorem 5.

THEOREM 6. Let $A$ be any ring and $M$ an A-module. If $K \subseteq \operatorname{Rad} M$ and $K$ is a minimally generated and injective, then $K=\{0\}$.

Proof. Let $M=N \oplus K$. Since $M / N \approx K, M / N$ has a minimal generator set. But $M=N+\operatorname{Rad} M$, so $\operatorname{Rad}(M / N)=M / N$ and by Theorem $1, M=N$, so $K=\{0\}$. 
Corollary 3. If $M$ is a left module of a left perfect ring, $\operatorname{Rad} M$ does not contain a non-zero injective submodule.

Corollary 4. If $M$ has no proper maximal submodules, then $M$ does not contain a non-zero minimally generated, injective submodule.

\section{BIBLIOGRAPHY}

1. H. Bass, Finitistic dimension and a homological generalization of semi-primary rings, Trans. American Math. Soc. 95 (1960), 466-488. MR\# 1212.

2. J. Lambek, Lectures on rings and modules, Waltham, Massachusetts, Toronto, London, Blaisdell Publishing Company, 1966.

3. B. T. Osofsky, A generalization of quaisi-frobenius rings, J. Algebra 4 (1966), 373-387, MR\# 4305.

4. W. H. Rant, Left perfect rings that are right perfect and a characterization of Steinitz rings, Proceedings of American Math. Soc., Volume 32, Number 1, March, 1972.

5. P. Ribenboim, Rings and modules, New York, London, Sydney, Toronto, Interscience Publishers, 1969.

Department of Natural Sciences Mathematics

LINCOLN UNIVERSITY, 1978

JEFFERSON CITY, MisSOURI 65101 